\title{
Gestão do Conhecimento Pessoal como potencializadora da geração de ideias: estudo sobre um programa de reconhecimento por geração de ideias em empresa de consultoria.
}

\author{
Alexandre Ferreira Nascimento \\ Mestrando em Gestão e Estratégia pelo Programa de Mestrado Profissional em Gestão e Estratégia da \\ Universidade Federal Rural do Rio de Janeiro. \\ nascimaa@gmail.com
}

Américo da Costa Ramos Filho

Doutor em Administração pela Escola Brasileira de Administração Pública e de Empresas da Fundação Getúlio Vargas. Professor do Departamento de Administração da Universidade Federal Fluminense. Professor do

Programa de Mestrado Profissional em Gestão e Estratégia da Universidade Federal Rural do Rio de Janeiro. americoramos@id.uff.br

André Yves Cribb

Doutor em Engenharia de Produção pela Universidade Federal do Rio de Janeiro. Professor do Programa de Mestrado Profissional em Gestão e Estratégia da Universidade Federal Rural do Rio de Janeiro. andre.cribb@embrapa.br

\section{Resumo}

O estudo tem como objetivo descrever a contribuição da gestão do conhecimento pessoal GCP para a geração de ideias inovadoras. A partir da análise de um framework sobre as dimensões do conhecimento pessoal como um fenômeno desenvolvido de forma sistemática e natural, é possível confirmar sua capacidade de potencializar o processo de geração de ideias. Em paralelo, investigou-se o programa de reconhecimento por geração de ideias em uma empresa de consultoria com o intuito de verificar em quais dimensões da GCP as ideias surgem. O estudo é de natureza qualitativa, de caráter descritivo, desenvolvido por meio de pesquisa bibliográfica, documental e estudo de caso simples. Os dados foram coletados através de entrevista semiestruturada com os empregados que tiveram suas ideias premiadas nos últimos dois anos. A análise dos dados ocorreu por meio dos métodos de análise indutiva, categorização e codificação descritiva, assim como o método de emparelhamento. Os resultados da pesquisa apontam que o conhecimento individual é relevante na medida em que o acúmulo de experiências profissionais permite ao empregado propor melhorias nas atividades e rotinas de trabalho, ao passo que o conhecimento oriundo das comunidades e redes permite ao empregado propor soluções para problemas de negócio ou gerar novos serviços. É possível concluir que a GCP potencializa a geração de ideias na dimensão individual, na forma de recuperação e aplicação do conhecimento para a sugestão de ideias, e na dimensão relacional, em redes ou comunidades, pela capacidade de atribuição de sentido ao conhecimento existente que servem de base para novas experiências e conhecimentos.

Palavras-Chave: Gestão do conhecimento pessoal. Geração de ideias. Programa de Recompensas.

\begin{abstract}
The aim of this study is to describe the contribution of personal knowledge management PKM for generating ideas. Considering the framework regarding the dimensions of personal knowledge, the purpose is to present the knowledge management as a phenomenon developed in a systematic and natural way through learning and social networks. Therefore it is possible to confirm their capacity to enhance the generation ideas process. In addition, the recognition program for generating ideas in a consulting firm we investigated in order to verify which
\end{abstract}


dimensions of PKM the ideas are arise. The study is classified as qualitative research and descriptive approach, developed through bibliography, documentary and case study methodologies. The data were collected through semi-structured interviews with employees who had their ideas rewarded in the past two years. The analysis was carried out through the methods of inductive analysis, categorization and descriptive coding, as well as the pairing method. The results indicate that individual knowledge is relevant to the extent that the accumulation of professional experience enables employees to propose improvements in their activities and work routines, while for the knowledge from social networks enables employees to propose solutions to business issue or generate new services. The results points out that PKM enhances the generation ideas in the individual perspective through the recovery and knowledge application, and in the social networks perspective or communities across allocation meaning the existing knowledge used as basis for new experiences and knowledge.

Keywords: Personal Knowledge Management. Create Ideas. Recognition Program.

\section{1 - INTRODUÇÃO}

A evolução da sociedade através da utilização de tecnologias e meios de comunicação avançados proporciona um convívio em redes de múltiplas conexões, contribuindo para o aumento da demanda por respostas rápidas e assertivas. Desta forma, empresas que buscam crescimento sustentável com lucratividade precisam intensificar seus esforços no desenvolvimento de métodos de gestão e ferramentas tecnológicas para potencializar sua capacidade competitiva e garantir seu poder de influência no mercado (NASCIMENTO; ENTRIEL, 2014). Portanto, é preciso agir estrategicamente investindo tempo e recursos em inovação contínua, de forma a sustentar o crescimento com lucratividade.

Um dos métodos de gestão de maior importância nas organizações a partir da década de 1990 é a gestão do conhecimento - GC (OLIVEIRA, 2009), que visa contribuir para o alcance da efetividade organizacional por meio de suas amplas possibilidades de processamento, armazenamento e disseminação do conhecimento, amparados nas tecnologias de informação e comunicação - TIC (WEN, 2009). A GC é considerada um ativo intangível nas organizações e se torna um diferencial competitivo pela capacidade de promover mudança conceitual e comportamental, transformando o conhecimento em competência por meio do acúmulo de experiências individuais. Por este motivo, a gestão do conhecimento pessoal - GCP torna-se um importante elemento potencializador da geração de ideias e inovação pela sua capacidade de auxiliar os indivíduos a se tornarem mais eficientes e produtivos frente às mudanças ambientais, tanto a nível pessoal quanto organizacional (PAULEEN; GORMAN, 2011).

A geração de ideias nas organizações sofre influência direta do conhecimento acumulado e das características dos indivíduos (GOFFIN; MITCHELL, 2005). Ademais, a geração de ideias alimenta os processos de inovação na medida em que as organizações transformam ideias em bens, serviços, novos processos ou melhorias significativas dos processos existentes, com o objetivo de progredir, competir ou diferenciar-se no mercado (BAREGHEH et al, 2009). Portanto, o processo de geração de ideias está vinculado à capacidade criativa do indivíduo para identificar problemas, gerar possíveis soluções e escolher aquela mais adequada ao contexto (NUNES, 2011). Consequentemente, as iniciativas sobre geração de ideias inovadoras devem focar seus esforços nas pessoas, uma vez que é por meio dos valores, pressupostos, experiências, conhecimento e interações no ambiente organizacional que elas pensam, agem, criam e inovam (MOREIRA; STRAMAR, 2014). 
Assumindo que a gestão do conhecimento pessoal favorece uma maior sensibilidade e disciplina do indivíduo, que contribui para a geração de ideias promovendo um ambiente propício a inovação, surgiu a pergunta que norteou este trabalho: como a gestão do conhecimento pessoal contribui com a geração de ideias inovadoras?

O objetivo deste estudo é descrever a contribuição da GCP para a geração de ideias inovadoras. Como objetivo intermediário, a pesquisa irá analisar um programa de recompensa por geração de ideias existente em uma empresa de consultoria à luz do modelo de GCP proposto por Efimova (2005).

Este artigo está estruturado com mais cinco seções além desta introdutória. A seção 2, a seguir, trata de uma abordagem teórica sobre os conceitos de gestão do conhecimento nos âmbitos organizacional e pessoal e geração de ideias. Na seção 3 será apresentada a metodologia de pesquisa. A seção 4 apresenta a empresa estudada, descreve o programa de geração de ideias abordado e demonstra a análise dos dados. A seção promove discussões à luz da literatura. Por fim, a seção 6 é destinada às considerações finais.

\section{2 - ARCABOUÇO TEÓRICO}

\section{1 - Gestão do conhecimento}

Gestão do conhecimento - GC pode ser amplamente definida como o processo de aquisição, codificação, disseminação e simplificação do conhecimento por todas as áreas da organização de forma sistemática com objetivo de atender aos requisitos organizacionais e estratégicos (ABILI et al, 2011; FROST, 2014). Na visão de Cribb (2010), a GC precisa ser articulada e intencional a partir de práticas e processos embutidos nos indivíduos, grupos e estruturas físicas, compreendendo os aspectos humanos e tecnológicos. Oliveira et al (2016) propõem que a GC depende do contexto sociocultural e que sua essência é compartilhar os conhecimentos individuais para formar o conhecimento organizacional. Ramos Filho (2011) corrobora com esta visão ao afirmar que as empresas precisam consolidar as experiências pessoais e organizacionais acumuladas para desenvolver processos de aquisição de ativos intangíveis integrados às práticas de GC. Por sua vez, o desafio das organizações passa a ser a adoção de novas estruturas com o intuito de superar as barreiras de inovação e gerar conhecimento por meio do aprendizado (SÁ et al, 2013).

O propósito da GC nas organizações é melhorar o fluxo de conhecimento entre as pessoas, e também entre as pessoas e as organizações (BAILEY; CLARK, 2001). Para Wiig (2000), a GC é a sistemática e explícita gestão de atividades práticas, programas e políticas da organização relacionadas ao conhecimento. Portanto, identificar o conhecimento existente e utilizá-lo como instrumento de penetração no ambiente de negócios capacita as organizações para competirem no mercado (LANG, 2001; JUNG et al, 2003). Uma das teorias mais sólidas sobre criação e gestão do conhecimento organizacional - GCO é denominada espiral do conhecimento (NONAKA; TAKEUCHI, 1997) e engloba as dimensões epistemológica e ontológica. Os autores afirmam que o fundamento epistemológico é a distinção entre o conhecimento tácito e explícito, cuja chave para criação do conhecimento está na mobilização e conversão do conhecimento tácito. A dimensão ontológica, representada pelos níveis individual, grupo, organizacional e interorganizacional, é responsável pela criação do conhecimento, ficando a cargo da organização o dever de fomentar a criação desse conhecimento. 
O conhecimento tácito está relacionado à individualidade, às experiências adquiridas e está atrelado a fatores intangíveis do cotidiano, podendo ser classificado como único, cuja transmissão ocorre por meio de diálogos, narrativas e experiências guiadas (LINS, 2003; LEONARD; SWAP, 2005). Já o conhecimento explícito é formal, fácil de ser transmitido, podendo ser codificado em fórmulas, especificações e regras (LEITE; COSTA, 2007). O conhecimento explícito está mais próximo do conhecimento científico uma vez que é repassado através de um aprendizado didático (MONTENEGRO JUNIOR, 2012).

Neste contexto, é possível afirmar que a GCO ocorre tanto na dimensão organizacional quanto na dimensão pessoal, uma vez que configura-se como fonte de fortalecimento e criação do conhecimento de forma dinâmica e relacional por meio da aprendizagem contínua, contribuindo para que as pessoas consigam expandir suas capacidades de gerar resultados (EASTERBY-SMITH; BURGOYNE, 2003; SENGE, 2006). De acordo com Azma e Mostafarpour (2011), a GCO envolve criatividade e inovação constante, além da gestão da aprendizagem coletiva e individual.

\section{2 - Gestão do conhecimento pessoal}

Gestão do conhecimento pessoal - GCP configura-se como uma forma sofisticada dos indivíduos gerenciarem suas vidas pessoais e profissionais (PAULEEN; GORMAN, 2011). GCP é comumente definida como o desenvolvimento de um conjunto de competências, habilidades e comportamentos que permite ao indivíduo prosperar em ambientes organizacionais e sociais turbulentos, complexos e em constante mudança por meio da aplicação assertiva do conhecimento (JUNG, 2009; ZHEN et al, 2012).

Neste contexto, a GCP deve preocupar-se com a investigação de como os indivíduos podem melhorar sua produtividade através de uma efetiva e consciente codificação do conhecimento pessoal para que possa ser reutilizado constantemente (VÖLKEL; ABECKER, 2008). Para Agnihotri e Troutt (2009), o propósito da GCP é auxiliar o individuo a gerenciar seu próprio conhecimento de forma que possa ser armazenado, selecionado, recuperado e compartilhado nas atividades diárias de trabalho e principalmente na solução de problemas e geração de ideias. Portanto, a GCP pode trazer vantagens tanto para o indivíduo quanto para a organização, possibilitando a identificação das lacunas e de habilidades pessoais, além da disseminação do conhecimento interno (JAIN, 2011). Wright (2012) propõe que a GCP deve fornecer um caminho estruturado para que os indivíduos possam constantemente avaliar suas próprias atividades com o objetivo de identificar seus pontos fortes e principais pontos de aprimoramento. Desta forma, a ênfase da GCP está direcionada para encorajar os indivíduos a refletirem sobre como estão trabalhando, tanto de forma individual quanto colaborativa, possibilitando o desenvolvimento de mecanismos para atuar com maior eficiência por meio de novas ideias.

Efimova (2005) propõe um framework com o intuito de exemplifica a relação indissociável entre a GCP e o processo de geração de ideias, com destaque para o potencial de contribuição mútua existente a partir de três dimensões: individual, comunidades e redes, e ideias. A autora afirma que esta abordagem multidisciplinar deve ser aplicada a GCP uma vez que permite a interação entre as três dimensões, partindo de uma perspectiva individual para uma coletiva através das relações em redes, cujo resultado é a geração de ideias. A proposta é que este framework seja aplicado em ambiente Web, uma vez que o conhecimento é desenvolvido naturalmente através das interações sociais, e que a Web é o ambiente ideal para o compartilhamento do conhecimento e o aumento das redes de relacionamento social (SNOWDEN et al, 2011). No entanto, Pauleen e Gorman (2011) ressaltam que a tecnologia 
da informação e comunicação - TIC é um importante instrumento que apoia a GCP de forma eficaz, porém, não pode ser vista como equivalente. As discussões sobre GCP englobam não apenas a perspectiva individual, mas também as relações pessoais e profissionais que conectam as pessoas que buscam cultivar e fortalecer seus conhecimentos pessoais. A figura 1 a seguir demonstra a relação proposta por Efimova (2005) entre indivíduos, comunidades e redes e geração de ideias.

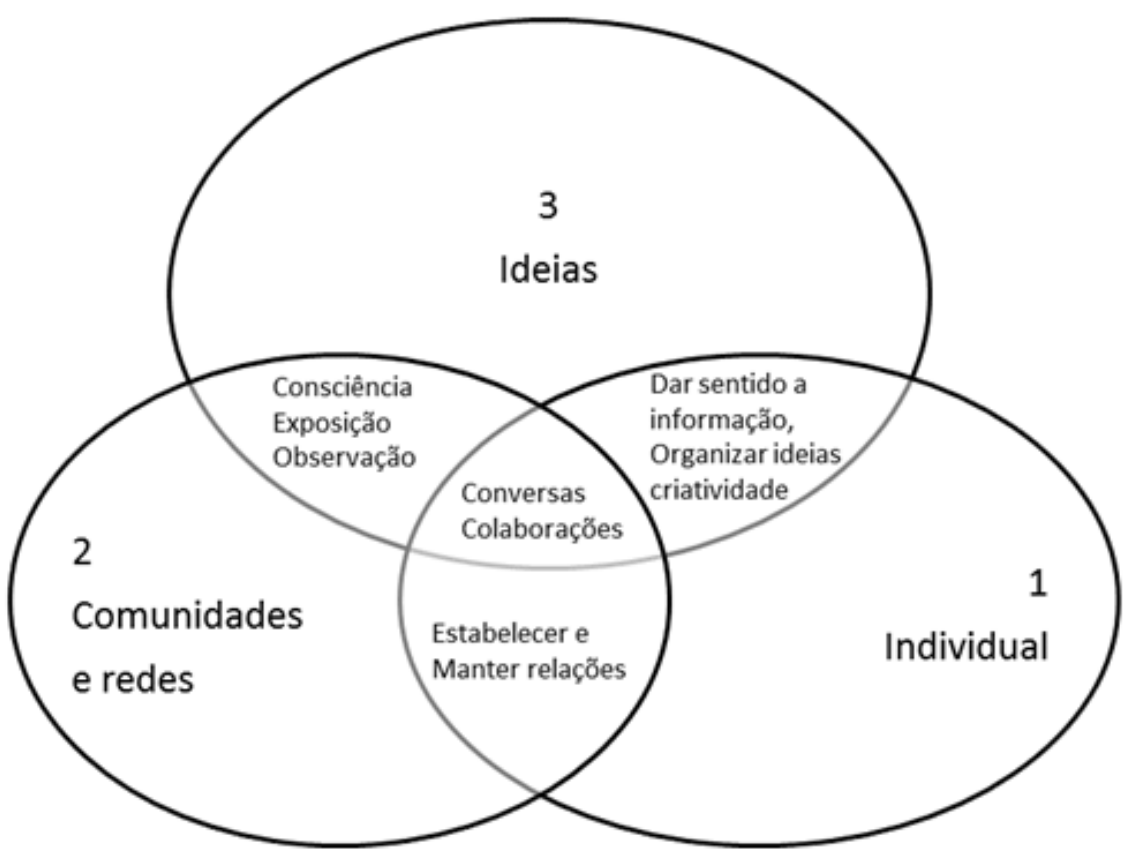

Figura 1 - Personal Knowledge Management Framework

Fonte: Dimensão do conhecimento organizacional (EFIMOVA, 2005).

Na visão de Smedley (2009), as competências e interações sociais apoiam as atividades de trabalho na formação de equipes para solução de problemas uma vez que promove um espaço de diálogo e reflexão. De forma complementar, Levy (2011) reconhece a importância da promoção das interações entre grupos de indivíduos ao introduzir um conceito social no contexto da GCP, destacando a relevância do incentivo às conversas coletivas. Neste sentido, o conhecimento pessoal é utilizado nas relações sociais através das comunidades e redes, o que potencializa a geração de ideias.

No que tange a geração de ideias, Barbieri et al (2009b) reforçam que todas as ideias interagem e se somam a outras geradas em diferentes fases dos processos de solução de problemas, desenvolvimento e implantação de um novo serviço ou produto. Por este motivo, serão apresentados a seguir os conceitos de geração de ideias.

\section{3 - Geração de ideias}

A geração de uma ideia, também denominada na literatura como ideação, corresponde à transformação da inteligência competitiva em ideias e insights que corroboram efetivamente com o aproveitamento das oportunidades identificadas (BORCHARDT; SANTOS, 2014). As ideias surgem em função de dois motivos básicos: (a) solucionar problemas relacionados com a produção e comercialização de bens e serviços; (b) aproveitar as oportunidades de ampliação dos conhecimentos gerados na empresa e no ambiente externo (BARBIERI et al, 2009a). Para os autores, uma ideia se expressa mediante uma opinião, ponto de vista, noção, conhecimento ou qualquer outro meio capaz de representar a concepção mental de algo 
concreto ou abstrato, transformando-se não apenas em representação mental de um objeto existente, mas também numa possibilidade ou antecipação de algo. De acordo com Dewes et al (2011) para tornar os ambientes mais favoráveis às inovações, é preciso desenvolver mecanismo de estímulo à criatividade por meio da geração de ideias.

Dijk e Ende (2002) afirmam que os programas de sugestões são uma importante forma de geração de ideias, pois canaliza as criatividades dos funcionários, bem como dá suporte à implantação de uma cultura de inovação. É possível encontrar na literatura especializada duas abordagens para os programas de sugestões. Uma delas considerada como um sistema tradicional, conhecido como caixa de sugestões, cuja origem é ocidental, caracterizando-se pela busca de ideias geniais estimuladas por recompensas econômicas. A outra abordagem está relacionada ao conceito Kaizen, baseada na contribuição voluntária de todos os funcionários, e cuja origem é oriental (BARBIERI et al, 2009a). Na primeira abordagem, a forma de recompensa é através de um prêmio em dinheiro ao proponente da ideia, desde que a mesma seja aprovada por comissões de análise antes de ser encaminhada para a direção ou alta administração da empresa, sempre com foco em ganhos econômicos. Já na segunda abordagem, as sugestões são recompensadas por premiações simbólicas, tais como estabilidade no emprego, oportunidade de crescimento profissional, homenagens públicas, cujo objetivo é reforçar a coesão interna e promover um ambiente de trabalho que aproveite as oportunidades de melhorias (BARBIERI et al, 2009a).

A partir de uma pesquisa realizada em três empresas brasileiras para análise dos modelos de geração de ideias, Barbieri et al (2009a) propõem um modelo sistematizado para captação de ideias denominado sistema de sugestões, com características de ambas abordagens citadas anteriormente, cujo propósito é envolver todos os colaboradores da organização, independentemente de sua área de atuação. Para os autores, o sistema de sugestões configurase como ferramenta estratégica uma vez que contempla as distintas visões dos empregados dentro da empresa.

O sistema de sugestões proposto por Barbieri et al (2009a) tem como objetivo estimular a geração de ideias dos empregados envolvidos em atividades específicas, o que requer a utilização do conhecimento acumulado nas rotinas de trabalho. $O$ sistema prevê a aprendizagem contínua por meio das experiências de trabalho e das relações entre as pessoas, entre os diversos setores das empresas e com o envolvimento das partes interessadas. Portanto, a geração de uma nova ideia, ou um novo conceito de trabalho, configura-se como processo crítico da inovação, uma vez que a ideia desperta a inovação, ao mesmo tempo em que não há inovação que não tenha surgido de uma ideia (NUNES, 2011).

\section{3 - METODOLOGIA}

A pesquisa é de natureza qualitativa, uma vez que tem por objetivo analisar e compreender dados textuais em contextos em que os participantes de um estudo abordam um problema ou questão concreta (CRESWELL, 2014). Os procedimentos técnicos adotados foram, quanto aos fins, pesquisa de caráter descritiva, pois identifica os fatores que determinam ou contribuem para a ocorrência de determinado fenômeno e aprofunda o conhecimento da realidade (GIL, 2007). Quanto aos meios, optou-se por pesquisa bibliográfica em livros e trabalhos científicos, uma vez que fornece instrumento analítico para todo tipo de pesquisa (VERGARA, 2013), pesquisa documental sobre o modelo de geração de ideias da empresa analisada com o intuito de recolher informações prévias sobre o campo de interesse (MARCONI; LAKATOS, 2010), e estudo de caso simples, que permite a investigação 
empírica de um fenômeno contemporâneo, utilizando-se da abrangência do acesso que o pesquisador teve sobre eventos comportamentais efetivos no contexto estudado (YIN, 2005).

Os dados foram coletados através de entrevistas semiestruturadas com roteiro elaborado por cinco questões abertas, que permitiram ao entrevistador entender e captar a perspectiva dos participantes da pesquisa (ROESCH, 1999, CRESWELL, 2014). O quadro 1 demonstra a caracterização dos sujeitos da pesquisa.

Quadro 1 - Caracterização dos sujeitos da pesquisa

\begin{tabular}{|c|c|c|c|c|c|}
\hline Matrícula & Idade & Gênero & Formação & Experiência profissional & Tempo na empresa \\
\hline 10777 & 40 & $\mathrm{M}$ & Atuária & $\begin{array}{c}\text { Previdência } \\
\text { complementar }\end{array}$ & 12 anos \\
\hline 18251 & 31 & $\mathrm{~F}$ & Estatística & $\begin{array}{c}\text { Previdência } \\
\text { complementar }\end{array}$ & 8 anos \\
\hline 20105 & 32 & $\mathrm{~F}$ & Direito & Legal and Compliance & 3 anos \\
\hline 21560 & 50 & $\mathrm{M}$ & Atuária & $\begin{array}{c}\text { Previdência } \\
\text { complementar }\end{array}$ & 1 ano \\
\hline 34798 & 44 & $\mathrm{~F}$ & Economia & Finanças & 1 ano \\
\hline
\end{tabular}

Fonte: Dados da pesquisa, 2016.

As entrevistas foram realizadas no escritório da empresa na Cidade do Rio de Janeiro entre os dias trinta de maio e seis de junho de 2016 com os empregados que tiveram suas ideias premiadas nos últimos dois anos, período compreendido entre 01/01/2014 a 31/12/2015, totalizando cinco pessoas. O tempo de trabalho dos entrevistados na empresa varia entre $1 \mathrm{e}$ 12 anos, todos com idade superior a 30 anos. Adicionalmente, utilizou-se observação participante, de caráter empírico, para preencher possíveis lacunas.

Para análise dos dados utilizou-se o método de análise indutiva (THOMAS, 2006), que é um procedimento sistemático para analisar dados qualitativos como característica de ser guiada por objetivos específicos de avaliação. Optou-se pela técnica de codificação descritiva (GIBBS, 2009), cujo propósito é identificar e registrar uma ou mais passagens de texto ou outros itens dos dados. Por fim, os dados foram confrontados com a literatura apresentada na seção 2 deste estudo por meio do procedimento de emparelhamento (LAVILLE; DIONNE, 1999), que consiste em associar os dados recolhidos a um ou mais modelos teóricos com a finalidade de compará-los.

A seguir serão apresentados o programa de reconhecimento estudado, os detalhes dos procedimentos adotados para análise dos dados, e a discussão dos resultados à luz da literatura utilizada nesta pesquisa.

\section{4 - O CASO ESTUDADO}

\section{1 - A empresa}

A empresa objeto de pesquisa é uma consultoria de origem americana, líder global em serviços profissionais relacionados às práticas de Recursos Humanos, análises de Riscos e Serviços Financeiros e corretagem de Seguros e Valores Mobiliários. Está estruturada globalmente de forma matricial, dividindo seus esforços gerenciais por segmentos de mercados e geografia. Enquanto os segmentos são responsáveis pela gestão de pessoas, qualidade e entrega dos trabalhos, as regiões são responsáveis pelo relacionamento com os 
clientes e abertura de novos negócios. Atualmente, a empresa está presente em 120 países e conta com cerca de 39.000 empregados. No Brasil, acumula mais de 30 anos de experiência no mercado e possui sete escritórios, sendo a matriz na cidade de São Paulo, com 441 empregados, filial na cidade do Rio de Janeiro, que conta com 161 empregados e demais filiais em Belo Horizonte, Curitiba e Porto Alegre totalizando 31 empregados. Seu nome será preservado por questões de confidencialidade.

\section{2 - O modelo de geração de ideias}

O modelo de geração de ideias estudado é denominado Programa de Reconhecimento, de âmbito global, e tem como principal objetivo recompensar, de forma individual ou coletiva, ideias que contribuam para a superação de obstáculos presentes nas atividades de trabalho, cujo resultado potencializa o poder competitivo da empresa e fortalece o ambiente colaborativo. O programa está estruturado em duas categorias, uma com premiação em dinheiro e outra com reconhecimento simbólico.

Referente à premiação em dinheiro, o foco é recompensar as ideias de forma individual ou em grupo, de acordo com o impacto e contribuição para solução dos problemas enfrentados pelos clientes ou propostas de novos negócios. Portanto, tem uma perspectiva voltada para o negócio em si. Todos os empregados podem participar do programa de premiação em dinheiro e os critérios de avaliação levam em consideração questões como: o impacto que a ideia teve na solução de um problema ou elaboração de um novo negócio; o grau de aplicabilidade da ideia; e o grau de abrangência, ou seja, sua aplicação em uma linha de negócio ou para toda a empresa, e ainda, se em um país ou região.

As ideias são enviadas através de um formulário eletrônico disponível na intranet da empresa. As avaliações são realizadas por comissões especiais de acordo com o assunto e o resultado é divulgado num prazo máximo de 30 dias. As premiações ocorrem sempre no último dia útil do mês em que os resultados foram apurados pela mesma comissão avaliadora.

As classificações para as premiações estão destacadas no quadro 2 a seguir.

Quadro 2 - Classificações de ideias para premiação em dinheiro

\begin{tabular}{|l|l|l|}
\hline Impressionante & $\begin{array}{l}\text { Adaptações no escopo de trabalho que geram resultados expressivos } \\
\text { como redução de custos ou melhorias na execução dos projetos de } \\
\text { clientes }\end{array}$ & $\begin{array}{l}\text { Urêmios entre } \\
\text { US\$50 e US } \$ 500\end{array}$ \\
\hline Excepcional & $\begin{array}{l}\text { Decisões baseadas em profundo conhecimento que geram resultados } \\
\text { excepcionais como incremento de valor agregado e/ou novos } \\
\text { negócios para a empresa }\end{array}$ & $\begin{array}{l}\text { Prêmios entre } \\
\text { US\$501 e US\$5000 }\end{array}$ \\
\hline
\end{tabular}

Fonte: Dados da pesquisa, 2016.

No que se refere ao reconhecimento simbólico, permanece a lógica de recompensar as ideias com algum impacto e contribuição, tanto individual quanto coletivo, sendo que o foco, neste caso, está voltado para uma perspectiva interna, cujas ideias contribuam para soluções de problemas internos enfrentados pelas equipes de trabalhos e em todas as áreas de atuação. Assim como na premiação em dinheiro, todos os empregados podem participar do programa e os critérios de avaliação levam em consideração questões como: o impacto que a ideia teve para a melhoria de uma atividade de trabalho ou solução de um problema que engloba uma ou mais áreas ao mesmo tempo; o grau de aplicabilidade da ideia; e o grau de abrangência, ou seja, se a aplicação ocorre em uma área específica ou em diversas áreas da empresa, e ainda, se envolve um país ou uma região. Neste caso, as ideias são apresentadas e discutidas diretamente com o gerente da área envolvida, ou até com uma ou mais equipes de trabalho, 
sendo que a avaliação e análise da aplicabilidade são realizadas no ato da conversa e o reconhecimento pode ocorrer na mesma hora ou no mesmo dia. As formas de reconhecimento simbólico estão destacadas na figura 2 a seguir.

Figura 2 - Formas de reconhecimento simbólico

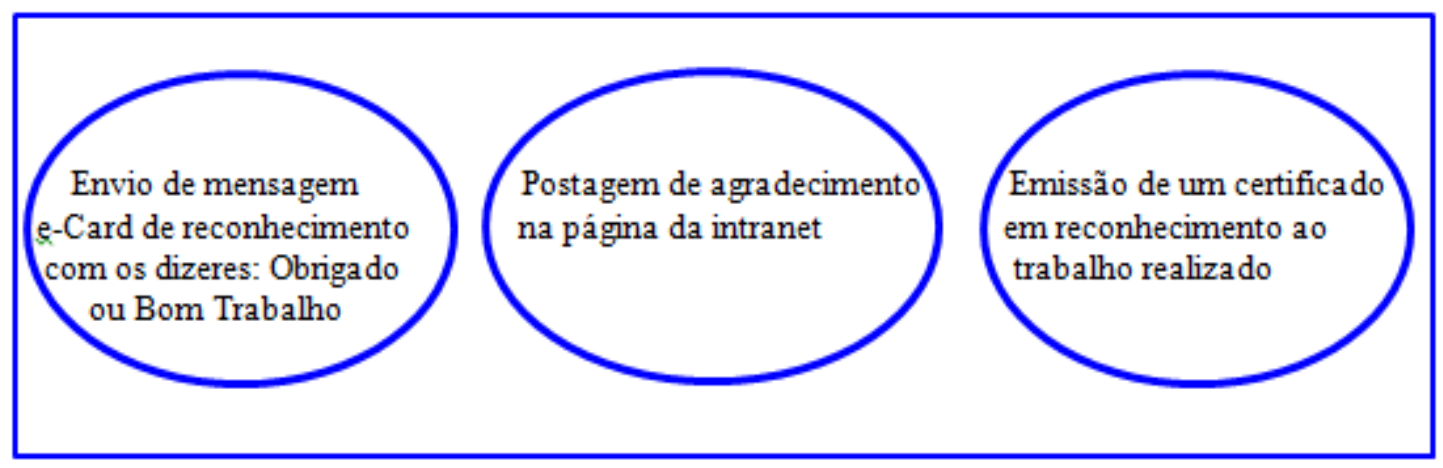

Fonte: Dados da pesquisa, 2016.

Não há qualquer diferenciação de importância ou impacto na escolha de uma das formas de reconhecimento citadas acima, ficando sob a inteira responsabilidade de o gerente utilizar aquela que mais se adeque ao perfil do empregado ou grupo, ou ainda, de acordo com o contexto e intensão de divulgação.

\section{3 - Análise dos dados}

O roteiro das entrevistas semiestruturadas foi elaborado com um total de cinco perguntas fundamentadas no framework proposto por Efimova (2005). A primeira pergunta foi relacionada à dimensão do indivíduo, as perguntas dois e três foram ligadas à dimensão das relações interpessoais por meio das comunidades e redes, e as perguntas quatro e cinco atreladas à geração de ideias. A transcrição de cada entrevista foi realizada na íntegra logo após sua realização.

A etapa de preparação dos dados obedeceu a seguinte sequência: (i) preparação dos arquivos de dados brutos para estruturá-los em formato comum; (ii) leitura atenta e detalhada do texto para compreensão dos temas abordados; (iii) criação de categorias a partir dos objetivos da pesquisa e de frases reais ou significado em segmento de texto específico; (iv) sobreposição de categorias e texto não codificado; (v) revisão e refinamento do sistema de categorias visando identificar sub tópicos dentro das categorias, selecionado citações que transmitam a ideia central da categoria.

O processo de análise foi realizado a partir das etapas categorização, codificação descritiva, além da análise comparativa, com o intuito de estabelecer relações entre os objetivos do estudo e as conclusões (THOMAS, 2006). Utilizou-se a técnica de codificação descritiva dos dados proposta por Gibbs (2009) com o propósito de identificar e registrar uma ou mais passagens de texto da transcrição das entrevistas estabelecendo uma relação com um nome ou uma ideia. Os resultados obtidos com a categorização e codificação dos dados foram analisados e confrontados com o arcabouço teórico e seus desdobramentos utilizando-se do método de emparelhamento proposto por Laville e Dionne (1999).

O quadro 3 a seguir demonstra um resumo claro e objetivo dos resultados dos processos de codificação, categorização e emparelhamento dos dados da pesquisa de campo com os conceitos apresentados na seção 2 proveniente do arcabouço teórico e seus desdobramentos. 
Quadro 3 - Síntese da codificação, categorização e emparelhamento.

\begin{tabular}{|c|c|c|c|}
\hline Código & Categorias & Descrição das categorias & Emparelhamento \\
\hline \multirow{2}{*}{ IND } & \multirow{2}{*}{ Indivíduo } & $\begin{array}{l}\text { O indivíduo se desenvolve a } \\
\text { partir da aprendizagem e } \\
\text { acúmulo de experiência } \\
\text { profissional e pessoal }\end{array}$ & $\begin{array}{l}\text { GCO ocorre na dimensão pessoal, e } \\
\text { configura-se como fonte de } \\
\text { fortalecimento e criação do } \\
\text { conhecimento de forma dinâmica e } \\
\text { relacional por meio da aprendizagem } \\
\text { contínua, contribuindo para que as } \\
\text { pessoas consigam expandir suas } \\
\text { capacidades de gerar resultados } \\
\text { (EASTERBY-SMITH; BURGOYNE, } \\
\text { 2003; SENGE, 2006). } \\
\text { GCP auxilia os indivíduos a se } \\
\text { tornarem mais eficientes e produtivos } \\
\text { frente às mudanças ambientais, tanto a } \\
\text { nível pessoal quanto organizacional } \\
\text { (PAULEEN; GORMAN, 2011). }\end{array}$ \\
\hline & & $\begin{array}{l}\text { O indivíduo contribui de forma } \\
\text { efetiva com a organização na } \\
\text { tomada de decisão, solução de } \\
\text { problemas e criação de ideias }\end{array}$ & $\begin{array}{l}\text { GCP auxilia o individuo a gerenciar } \\
\text { seu próprio conhecimento de forma } \\
\text { que possa ser compartilhado nas } \\
\text { atividades diárias de trabalho e } \\
\text { principalmente na solução de } \\
\text { problemas e geração de ideias } \\
\text { (AGNIHOTRI; TROUTT, 2009). } \\
\text { GCP é um conjunto de competências, } \\
\text { habilidades e comportamentos que } \\
\text { permite ao indivíduo prosperar em } \\
\text { ambientes organizacionais e sociais } \\
\text { turbulentos, complexos e em constante } \\
\text { mudança por meio da aplicação } \\
\text { assertiva do conhecimento (JUNG, } \\
\text { 2009; ZHEN et al, 2012). }\end{array}$ \\
\hline \multirow{2}{*}{ CO\&RE } & \multirow[b]{2}{*}{$\begin{array}{c}\text { Comunidades } \\
\text { e redes }\end{array}$} & $\begin{array}{l}\text { A colaboração e o diálogo } \\
\text { entre as pessoas e grupos de } \\
\text { pessoas promove um ambiente } \\
\text { de interação social }\end{array}$ & $\begin{array}{l}\text { As interações sociais apoiam as } \\
\text { atividades de trabalho na formação de } \\
\text { equipes para solução de problemas } \\
\text { uma vez que promove um espaço de } \\
\text { diálogo e reflexão (SMEDLEY, 2009). }\end{array}$ \\
\hline & & $\begin{array}{l}\text { A tecnologia elimina as } \\
\text { barreiras de comunicação } \\
\text { dentro da organização }\end{array}$ & $\begin{array}{l}\text { A tecnologia da informação e } \\
\text { comunicação - TIC é um importante } \\
\text { instrumento da GCP no apoio a criação } \\
\text { de um ambiente ideal para o } \\
\text { compartilhamento do conhecimento e } \\
\text { o aumento das redes de relacionamento } \\
\text { social (SNOWDEN et al, 2011), } \\
\text { porém, não pode ser vista como } \\
\text { equivalente (PAULEEN; GORMAN, } \\
\text { 2011). }\end{array}$ \\
\hline
\end{tabular}




\begin{tabular}{|c|c|c|c|}
\hline Código & Categorias & Descrição das categorias & Emparelhamento \\
\hline & & $\begin{array}{l}\text { O conhecimento aplicado } \\
\text { desenvolve novos serviços }\end{array}$ & $\begin{array}{l}\text { Não há inovação que não tenha } \\
\text { surgido de uma ideia (NUNES, 2011). } \\
\text { A geração de uma ideia corresponde à } \\
\text { transformação da inteligência } \\
\text { competitiva em ideias e insights } \\
\text { (BORCHARDT; SANTOS, 2014). }\end{array}$ \\
\hline IDE & Ideias & $\begin{array}{l}\text { As ideias são geradas em } \\
\text { ambientes colaborativos que } \\
\text { envolvem equipes } \\
\text { interdisciplinares }\end{array}$ & $\begin{array}{l}\text { Para tornar os ambientes mais } \\
\text { favoráveis às inovações, é preciso } \\
\text { desenvolver mecanismo de estímulo à } \\
\text { criatividade (DEWES et al, 2011). } \\
\\
\text { Todas as ideias interagem e se somam } \\
\text { a outras geradas em diferentes fases } \\
\text { dos processos de solução de } \\
\text { problemas, desenvolvimento e } \\
\text { implantação de um novo serviço ou } \\
\text { produto (BARBIERI et al, 2009b). }\end{array}$ \\
\hline
\end{tabular}

Fonte: Dados da pesquisa, 2016.

A síntese apresentada revela que, na visão dos entrevistados, existe uma simbiose entre o framework de GCP proposto por Efimova (2005) e a rotina de geração de ideias no Programa de Reconhecimento existente na empresa estudada, cujo propósito é promover um ambiente inovador e disseminar uma cultura de colaboração contínua e sistemática por meio da geração de ideias.

A partir dos conceitos estudados e da forma como o Programa de Reconhecimento está estruturado, é possível concluir que, para as formas de recompensas simbólicas, o conhecimento individual é mais relevante na medida em que o acúmulo de experiências profissionais permite ao empregado propor melhorias nas atividades e rotinas de trabalho. No caso das recompensas financeiras, que estão diretamente relacionados ao negócio e envolvem a solução de problemas ou a proposição de novos serviços, o conhecimento existente nas comunidades e redes nas quais o empregado está inserido torna-se mais importante devido à capacidade de interpretar contextos e perceber as mudanças no ambiente. Cabe ressaltar que as comunidades e redes de relacionamento não estão diretamente relacionadas com o uso de TIC como Efimova (2005) propõe na aplicação do framework de GCP.

\section{5 - DISCUSSÃO DOS RESULTADOS À LUZ DA LITERATURA}

Na primeira categoria classificada como Indivíduo, é possível destacar que, na visão dos entrevistados, o desenvolvimento pessoal ocorre por meio das experiências acumuladas permitindo maior contribuição na forma de solução de problemas e criação de ideias. A literatura sobre GCP destaca que a criação de conhecimento de forma dinâmica e relacional contribui para que as pessoas aumentem suas capacidades de gerar resultados, para que se tornem mais eficientes, produtivas e criativas para propor novas ideias e melhorias no trabalho (EASTERBY-SMITH; BURGOYNE, 2003; SENGE, 2006; PAULEEN; GORMAN, 2011) ao mesmo tempo em que permite o autogerenciamento e autonomia nas tomadas de decisões (AGNIHOTRI; TROUTT, 2009; JUNG, 2009; ZHEN et al, 2012). 
$\mathrm{Na}$ segunda categoria identificada como Comunidades e Redes, os principais destaques extraídos das respostas dos entrevistados são que a colaboração e o diálogo promovem um ambiente de interação social e que a tecnologia elimina as barreiras de comunicação na empresa. A literatura destaca que as interações sociais promovem um espaço de diálogo e reflexão na formação de equipes para a solução de problemas (SMEDLEY, 2009) e que a tecnologia é um importante instrumento de apoio para a GCP (SNOWDEN et al, 2011), porém, não pode ser vista como equivalente (PAULEEN; GORMAN, 2011).

$\mathrm{Na}$ terceira e última categoria identificada como Ideias, os principais achados a partir da interpretação das respostas são que o conhecimento aplicado desenvolve novos serviços e que os ambientes colaborativos promovem a geração de ideias entre equipes multidisciplinares. A literatura destaca que os ambientes favoráveis à inovação são aqueles que estimulam a criatividade (DEWES et al, 2011) e que as ideias interagem com outras geradas em diferentes fases de um mesmo processo (BARBIERI et al, 2009b) que necessariamente envolve o diálogo entre pessoas e grupos diferentes.

A pesquisa revelou que os empregados que tiveram suas ideias premiadas pelas duas formas de recompensa previstas no programa adquirem conhecimento por meio das experiências de trabalho e das interações sociais a partir dos diálogos com as equipes de trabalho. Foi possível perceber que as pessoas armazenam, recuperam e compartilham seus conhecimentos de maneiras diferentes. Enquanto os empregados que utilizam o conhecimento na dimensão individual demonstraram-se mais preocupados nas atividades diárias de trabalho e como podem crescer e prosperar individualmente, os empregados que utilizam suas redes e comunidades atribuem valor as atividades que envolvem o negócio da empresa em múltiplos processos, buscando o diálogo constante e trabalho em equipe com as diversas áreas da empresa.

Invariavelmente, os indivíduos utilizam suas experiências e conhecimentos acumulados para propor ideias ou soluções para os problemas que melhor se adequam as suas necessidades ou realidades. Portanto, identificar o conhecimento existente na organização através das ideias sugeridas fortalece o poder de penetração e competição da organização (LANG, 2001). Ademais, a GCP potencializa a geração de ideias uma vez que atribui sentido ao conhecimento existente, contribuindo para seu compartilhamento em grupos ou comunidades através das ideias (HIGGISON, 2004).

O framework proposto por Efimova (2005) demonstra as dimensões nas quais a GCP contribui com a geração de ideias pela sua abordagem multidisciplinar e pela capacidade de compartilhar o conhecimento em redes de relacionamento. Apesar de a autora propor que o framework deve ser aplicado em ambiente Web alegando a necessidade do apoio de tecnologia da informação e comunicação - TIC, o estudo demonstrou que o conhecimento é desenvolvido naturalmente nas relações e interações sócias presenciais e que a TIC configurase como elemento capaz de eliminar as barreiras de comunicação, mas não é considerada essencial para potencializando a geração de ideias (SNOWDEN et al, 2011; PAULEEN; GORMAN, 2011).

Diante do exposto, é possível concluir que a geração de ideias configura-se como forma sistematizada de identificação do conhecimento nas organizações e, ao mesmo tempo, contribui para a criatividade e desenvolvimento do indivíduo, uma vez que permite a recuperação e aplicação do conhecimento acumulado por meio da efetiva gestão do conhecimento pessoal. 


\section{6 - CONSIDERAÇÕES FINAIS}

Este artigo teve como objetivo descrever a contribuição da gestão do conhecimento pessoal para a geração de ideais inovadoras. O framework sobre gestão do conhecimento pessoal (EFIMOVA, 2005) extraído da literatura permitiu confrontar os conceitos de gestão do conhecimento pessoal com as premissas da geração de ideias, que é a recuperação e aplicação das experiências e conhecimentos acumulados, dentro de um determinado contexto, com o propósito de contribuir com ideias para o surgimento de um novo serviço ou produto, para solução de problemas existente no ambiente ou para melhoria de processos.

O estudo analisou o programa de geração de ideias existente na empresa e os resultados mostraram que a geração de ideias como processo sistematizado em múltiplos estágios contribui para o surgimento de bens, novos serviços ou melhorados, novos processos, além de potencializar a criatividade. É possível concluir que as ideias destinadas às melhorias nas rotinas de trabalho surgem na dimensão individual e as ideias destinadas à proposição de novos serviços ou solução de problemas de negócio surgem na dimensão das comunidades e redes. O estudo permitiu uma análise detalhada do framework sobre gestão do conhecimento pessoal (EFIMOVA, 2005) de forma que a abordagem multidisciplinar demonstra as dimensões nas quais a GCP contribui com a geração de ideias através da capacidade de aprendizagem individual e compartilhamento do conhecimento em redes de relacionamento. No entanto, foi não possível confirmar que o framework precisa ser aplicado em ambiente Web uma vez que demonstrou-se satisfatório sem utilização de sistema de informação.

Em resposta ao problema de pesquisa, após realização das análises das entrevistas, do programa de geração de ideias e dos conceitos de gestão do conhecimento, é possível afirmar que a GCP potencializa a geração de ideias na dimensão individual, por meio da recuperação e aplicação do conhecimento para a criatividade na sugestão das ideias e na dimensão relacional em redes ou comunidades, pela capacidade de contribuição mútua e atribuição de sentido ao conhecimento existente, gerando ideias inovadoras e servindo de base para novas experiências e conhecimentos.

Como contribuição prática, este estudo irá possibilitar que a empresa compreenda a relação entre a gestão do conhecimento pessoal e a geração de ideias, contribuindo para a melhoria contínua da captação e aproveitamento do conhecimento, transformando-o em ideias com potencial de gerar inovação. A contribuição acadêmica configura-se pelo fato da pesquisa enriquecer os conceitos de GCP e geração de ideias, apresentado análise entre a teoria extraída da literatura e a prática investigada na empresa.

Como proposta de futuros trabalhos, recomenda-se investigar como ocorre a geração de ideias especificamente nos processos de transferência de conhecimento tácito para verificar se as dimensões de contribuição do conhecimento se mostram ampliadas se comparadas às encontradas neste estudo.

\section{Referências}

ABILI, K.; et al. The role of effective Factors on Organizational Knowledge Sharing: International Conference on Education and Educational Psychology (ICEEPSY 2011). Procedia - Social and Behavioral Sciences 29. pp. 1701 - 1706, 2011. 
AGNIHOTRI. R.; TROUTT, M. D. The effective use of technology in personal knowledge management: a framework of skills, tools and user context. Online Information Review. v. 33, n. 2, p. 329-342, abr. 2009.

AZMA, F.; MOSTAFAPOUR, M. A. Identify knowledge management and organizational learning indicators and its relation with creativity. Social and Behavioral Sciences 30. 2011, $2249-2252$.

BAILEY, C.; CLARKE, M. Managing knowledge for personal and organizational benefit. Journal of Knowledge Management. 2001. Vol. $5 \mathrm{n}^{\mathrm{o}}$ 1, pp 58-67.

BARBIERI, J. C.; ÁLVARES, C. T.; CAJAZEIRA, J. E. R. Gestão de ideias para inovação contínua. Porto Alegre: Bookman. 2009a.

BARBIERI, J. C.; ÁLVARES, C. T.; CAJAZEIRA, J. E. R. GERAÇÃO DE IDEIAS PARA INOVAÇÕES: ESTUDOS DE CASOS E NOVAS ABORDAGENS. Revista Gestão Industrial. Universidade Tecnológica Federal do Paraná - UTFPR. Campus Ponta Grossa Paraná - Brasil. ISSN 1808-0448 / v. 05, n. 03: p. 01-20, 2009 b.

BAREGHEH, A.; ROWLEY, J.; SAMBROOK, S. Towards a multidisciplinary definition of innovation. Management Decision, 47(8), 1323-1339. 2009.

http://dx.doi.org/10.1108/00251740910984578

BORCHARDT, P.; SANTOS, G. V. GESTÃO DE IDEIAS PARA INOVAÇÃO: TRANSFORMANDO A CRIATIVIDADE EM SOLUÇÕES PRÁTICAS. RAI - Revista de Administração e Inovação. ISSN: 1809-2039. 2014. doi: 10.5773/rai.v11i1.11852014

CRESWELL, J. W. Investigação qualitativa e projeto de pesquisa: escolhendo entre cinco abordagens. $3^{\text {a }}$ Ed. - Porto Alegre: Penso, 2014.

CRIBB, A. Y. Uma Abordagem Pragmática de Construção de Estratégias de Gestão do Conhecimento em Organizações. Revista Iberoamericana de Sistemas, Cibernética e Informática, v. 7, p. 75-80, 2010.

DEWES, F.; NEVES, F. M.; JUNG, C. F.; CATEN, C. S. Ambientes e estímulos favoráveis à criatividade aplicada a processos de inovação de produtos. Anais. $8^{\circ}$ Congresso Brasileiro de Gestão de Desenvolvimento de Produto. Porto Alegre, 2011.

DIJK, C. van; ENDE, J. van den. Sugestion systems: transferring employee creativity into practicable ideas. Massachusetts (USA): R\&D Management, 32, 5, 2002.

EFIMOVA, L. Understanding personal knowledge management: A weblog case. Enschede: Telematica Instituut, 2005.

EASTERBY-SMITH, M., \& BURGOYNE, J. Introduction: Watersheds of Organizational Learning and Knowledge Management. In: EASTERBY-SMITH, M., \& BURGOYNE, J. (Org.). The Blackwell handbook of organizational learning and knowledge management. Malden/USA, 2003, p. 1-15.

FROST, A. A Synthesis of Knowledge Management Failure Factors. 
Disponível em: www.knowledge-management-tools.net/failure.html. Acesso em: 09/02/2016.

GIBBS, G. Análise de dados qualitativos. Porto Alegre: Artmed, 2009.

GIL, A. C. Como elaborar projetos de pesquisa. São Paulo: Atlas, 2007.

GOFFIN, K.; MITCHELL, R. Innovation management: Strategy and implementation using the pentathlon framework. London. Palgrave Mcmillan. 2005.

HIGGISON, S. Your say: Personal knowledge management. Inside Knowledge, 2004.

JAIN, P. Personal knowledge management: the foundation of organizational knowledge management. SA Jnl Libs \& Info Sci, 2011.

JUNG, D. I.; CHOW, C.; WU, A. The role of transformational leadership in enhancing organizational innovation: hypotheses and some preliminary findings, The Leadership Quarterly, 2003. Vol. 14 nº 20, pp. 525-544.

JUNG, J. J. Knowledge distribution via shared context between blog-based knowledge management systems: A case study of collaborative tagging. Expert Systems with Applications 36 (2009). Elsevier. 10627-10633.

LANG, J. C. Managerial concerns in knowledge management. Journal of knowledge management, Vol, 5. $\mathrm{n}^{\mathrm{o}} 1$. pp 43-57, 2001.

LAVILLE, C.; DIONNE, J. A construção do saber: manual de metodologia da pesquisa em ciências humanas. Belo Horizonte: Ed. UFMG, 1999.

LEITE, F.; COSTA, S. M. de S. Gestão do conhecimento científico: proposta de um modelo conceitual com base em processos de comunicação científica. Ciência da Informação, Brasília, v. 36, n. 1, p. 92-107, jan./abr. 2007.

LEONARD, D.; SWAP, W. Deep Smarts: How to Cultivate and Transfer Enduring Business Wisdom. Harvard Business School Press. 2005.

LEVY, P. The semantic sphere 1: computation, cognition, and information economy. ISTE Ltd and John Wiley \& Sons, Inc. 2011.

LINS, S. Transferindo conhecimento tácito: uma abordagem construtivista. Rio de Janeiro: E-Papers, 2003.

MARCONI, M. A.; LAKATOS, E. M. Fundamentos de Metodologia Científica. 7. Ed. São Paulo: Atlas, 2010.

MONTENEGRO JUNIOR, C. A. A importância da Gestão do Conhecimento: um estudo de caso realizado em duas organizações prestadoras de serviço. Revista ESAB. 2012.

MOREIRA, E.; STRAMAR, A. Modelo holístico da gestão da inovação com ênfase na cooperação, flexibilidade e adaptação. Revista de Administração e Inovação, São Paulo, v. 11, n.4, p.195, out./dez. 2014. 
NASCIMENTO, A. F.; ENTRIEL, A. L. OS FATORES CRÍTICOS DE SUCESSO PARA A IMPLANTAÇÃO DA GESTÃO POR PROCESSOS: UM ESTUDO DE CASO EM EMPRESA DE CONSULTORIA. Anais. X Congresso Nacional de Excelência em Gestão Niterói, 2014.

NONAKA, I.; TAKEUCHI, H. Criação de conhecimento na empresa: Como as empresas japonesas geram a dinâmica da inovação. Rio de Janeiro: Campus, 1997.

NUNES, C. V. Princípios de Construção para Geração de Ideias nas Organizações: Proposta e Verificação de um Framework. Dissertação (Mestrado em Engenharia de Produção). Programa de Pós-Graduação em Engenharia de Produção. COPE - Universidade Federal do Rio de Janeiro, 2011.

OLIVEIRA, R. B. C. Uma metodologia de modelagem de processos de negócio orientada à gestão da informação e do conhecimento. Dissertação (mestrado) - Universidade Federal de Minas Gerais, Escola de Ciência da Informação. 2009. Referências: f. 123-126

OLIVEIRA, R. R.; OLIVEIRA, R. R.; LIMA, J. B. Reflexão sobre a relação entre a mudança de cultura organizacional e a gestão do conhecimento. Perspectiva em Gestão \& Conhecimento, João Pessoa, v. 6, n. 1, p. 19-35, jan/jun. 2016.

PAUlEEN, D.; GORMAN, G. E. Personal Knowledge Management. Gower Publishing, Farnham. 2011. 269 pp.

RAMOS FILHO, A. C. Conhecimento e crescimento em organizações globais. Adm. MADE (Universidade Estácio de Sá), v. 15, p. 23-37, 2011.

ROESCH, S. M. A. Projetos de Estágios e de Pesquisa em Administração. São Paulo:

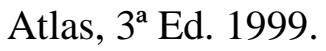

SÁ, F. B.; BENTO, K. G. R.; ZIVIANI, F.; FERREIRA, M. A. T. Práticas de Gestão do Conhecimento: Um estudo em organizações Mineiras. Perspectiva em Gestão e Conhecimento, João Pessoa, v. 3, n. 1, p. 114-131, jan/jun. 2013.

SENGE, P. M. A quinta disciplina: arte e prática da organização que aprende. Tradução: OP Traduções. 22. ed. Rio de Janeiro: Best Seller, 2006. 443 p.

SMEDLEY, J. K. Modelling Personal Knowledge Management. OR Insight (Dec 2009) Vol. 22, 4, 221-233.

SNOWDEN, D.; PAULEEN, D. J.; VAN VUUREN, S. J. Knowledge management and the individual: it's nothing personal. In: PAULEEN, D. J.; GORMAN, G. E.(Org.) Personal Knowledge Management: individual, organizational and social perspectives. Farnham Surrey: Gower Publishing Limited, 2011.

THOMAS, D. R. A general inductive approach for analyzing qualitative evaluation data. American Journal of Evaluation. v. 27, n. 2, Jun. 2006. 
VERGARA, S. C. Projetos e Relatório de Pesquisa em Administração. São Paulo: Atlas, 2013.

VÖLKEL, M; ABECKER, A. Cost-Benefit Analysis for the Design of Personal Knowledge Management Systems. Proc. of 10th International Conference on Enterprise Information Systems, Juni, 2008.

ZHEN, L.; SONG, H. T.; HE, J. T. Recommender systems for personal knowledge management in collaborative environments. Expert Systems with Applications 39. Elsevier. 2012. pp. 12536-12542.

YIN, R. K. Estudo de caso: Planejamento e Métodos. Porto Alegre: Bookman, 2005.

WEN, Y. F. An effectiveness measurement model for knowledge management. KnowledgeBased Systems 22. Elsevier. 2009. pp 363-367.

WIIG, K. M. Application of Knowledge Management in Public Administration. Texas, USA, 2000.

WRIGHT, K. Personal knowledge management: Planning Guide. Knowledge Resources. 2012. 